\title{
Electron impact ionisation of small molecules at intermediate energies: the molecular R-matrix with pseudostates method
}

\author{
J. D. Gorfinkiel and J. Tennyson \\ Department of Physics and Astronomy, University College London, Gower Street, London \\ WC1E 6BT, UK
}

\begin{abstract}
.
A procedure for the ab initio study of electron-molecule collisions at intermediate energies is presented in detail. The molecular $R$-matrix with pseudostates method (MRMPS) is based on the inclusion of discretized continuum states in the close-coupling expansion. This method allows, for the first time, the calculation of totally ab initio cross sections for electron impact ionisation of molecules as well as for electronic excitation above the first ionisation threshold. The method is general and can be applied to multielectron targets. Results for collisions with $\mathrm{H}_{3}^{+}$and $\mathrm{H}_{2}$ are presented. Numerical considerations necessary for performing a successful calculation are detailed.
\end{abstract}

PACS numbers:

\section{Introduction}

The theoretical study of electron molecule collisions has developed greatly over the last 20 years. Low-energy processes (rotational, vibrational or electronic excitation, dissociative recombination and attachment, electronic excitation to low lying states, etc.) are regularly investigated for small molecular targets. Their study is not without problems, but a variety of well tested approaches such as the R-matrix method, the complex Kohn variational method and the Schwinger multichannel method, are available (Huo and Gianturco 1995). At the high end of the energy range, perturbative methods can be successfully applied to the study of electronic excitation and ionisation.

In contrast, the intermediate energy regime has remained virtually untouched. This regime straddles the first ionisation threshold and extends to a few hundred of eV. Perturbative methods are not valid and $a b$ initio methods based on a close-coupling expansion (such as the R-matrix and complex Kohn variational methods) require, in principle, the inclusion of an infinite number of states. For a complete description of the electronic part (that is, neglecting nuclear motion) of electron-molecule collisions in the gas phase, successful treatment of this regime is a fundamental step. A few semi-empirical methods, available for determining the ionisation cross section in electron-molecule collisions, have been applied at intermediate energies. These include the Binary encounter Bethe (Kim and Rudd 1994) and 
its improvements (Huo 2001) and the Deustch Märk method (Deutsch et al. 2000). These methods provide a simple way of estimating the cross sections for ionisation, but are unable to provide information about excitation to bound states.

The same difficulties arise in the study of electron collisions with atoms at intermediate energy. It is only in the last 10 years that methods have been proposed and applied to near threshold ionisation. Indeed, only very recently has agreement been achieved for electron impact ionisation cross sections of $\mathrm{H}$. For simple targets, the ab initio Convergent Close-Coupling method (Bray et al. 2002) and Intermediate Energy R-matrix method (Burke et al. 1987) have proved highly successful. More recently, other methods have been developed, among which the most significant are the R-matrix with pseudostates (RMPS) (Bartschat et al. 1996), Exterior Complex Scaling (Rescigno et al. 1999) and Time-dependent Close-Coupling (Pindzola and Robicheaux 2000) procedures. Of these, only the RMPS method has been successfully applied to a variety of single and many electron targets.

We have developed an RMPS procedure to treat the general electron-molecule collision problem at intermediate energies as part of the UK R-matrix polyatomic code (Morgan et al. 1998). This method is based in the use of a set of states, known as pseudostates, that are not true eigenstates of the systems but that represent a discretized version of the continuum. Pseudostates have been used in collision studies for more than 30 years as a way of completing the close-coupling expansion not only in electron and positron-atom collisions but also in ion-atom ones. However, no systematic use of pseudostates for molecular systems has been reported. Our implementation, that we call molecular RMPS or MRMPS differs significantly from the atomic one. What simplifies the treatment in the atomic case (although it remains extremely complicated) is the fact that the continua are single-centred. Molecular continua are much more difficult to represent due to the lower symmetry and multicentre nature of the interaction potential.

In a previous letter (Gorfinkiel and Tennyson 2004), we reported results for $\mathrm{H}_{3}^{+}$and gave an outline of the method. $\mathrm{H}_{3}^{+}$is the smallest polyatomic ion and, more significantly, the dominant one in low-temperature hydrogenic plasmas. It plays a fundamental role in interstellar chemistry and has been observed in planetary aurora and diffuse interstellar media (McCall et al. 1998) where significant populations of energetic electrons are to be found. The interaction of $\mathrm{H}_{3}^{+}$with thermal (McCall et al. 2003, Kokoouline and Greene 2003) and higher energy (Kalhori et al. 2004) electrons remains an active area of study. Calculations of rotational excitation (Faure and Tennyson 2002a) and electronic excitation for energies up to $20 \mathrm{eV}$ are available (Orel 1992, Faure and Tennyson 2002b). However, there is no published information about collisions with intermediate energy electrons, although such experiments have been performed (Ghazaly and et al in preparation).

More recently, we have performed some initial calculations for $\mathrm{H}_{2}$. This is a benchmark system and a variety of experimental and theoretical data is available, not only for rotational, vibrational and electronic excitation processes but also ionisation, electron impact dissociation and dissociative recombination as well as detailed studies of its resonances (for a compilation of recent work on $\mathrm{H}_{2}$ see Brunger and Buckman (2002)).

In this paper, we present a detailed and complete description of the MRMPS method 
(section 2). In section 3 we present the results for $\mathrm{H}_{3}^{+}$; section 4 presents our preliminary results for $\mathrm{H}_{2}$ and the conclusions are outlined in Section 5.

\section{Method}

\subsection{Standard Fixed-Nuclei R-matrix method}

The application of the $R$-matrix method to polyatomic molecules within the fixed-nuclei approximation has been described in detail elsewhere (Morgan et al. 1997, Morgan et al. 1998, Tennyson and Morgan 1999). Briefly, the standard $R$-matrix method is based on dividing configuration space into two regions. The boundary between these regions is defined by a sphere of a given radius $a$ centred at the centre of mass of the molecule. In the inner region, exchange and electron correlation are taken into account using rigorous quantum chemistry methods. In the outer region, where these effects are negligible, the use of a longrange multipole potential suffices to describe the electron-molecule interaction. When the electronic part of the problem is very complex (as is the case at intermediate energies) Rmatrix calculations are so far restricted to the use of the fixed-nuclei (FN) approximation. In this approximation, the nuclear motion is neglected and the electronic wavefunctions are calculated at the ground state equilibrium geometry of the molecule.

In the inner region, the basis state wavefunctions for the $\mathrm{N}$-electron target + electron system are expanded in the following way:

$$
\Psi_{k}^{N+1}=\mathcal{A} \sum_{i j} a_{i j k} \phi_{i}\left(x_{1} \ldots x_{N}\right) u_{i j}\left(x_{N+1}\right)+\sum_{i} b_{i k} \chi_{i}\left(x_{1} \ldots x_{N+1}\right)
$$

where $\mathcal{A}$ is the antisymmetrization operator, $u_{i j}\left(x_{i}\right)$ are continuum orbitals and $x_{i}$ are the spatial and spin coordinates of electron $i, \phi_{i}$ are target wavefunctions, which are expressed in terms of a configuration interaction (CI) expansion and $\chi_{i}$ are known as $L^{2}$ functions. These $\chi_{i}$ are multi-centre quadratically integrable functions constructed from the target occupied and virtual molecular orbitals (MOs) and are used to represent correlation and polarization effects. They should be carefully chosen: the target wavefunctions and the $\mathrm{N}+1$ wavefunctions must be of equivalent quality. The wrong choice of $L^{2}$ functions can lead to overcorrelation of the $\mathrm{N}+1$ wavefunctions and an unbalanced calculation (for a discussion of this problem see Tennyson 1996).

In the polyatomic R-matrix suite, both the molecular and the continuum orbitals are expanded in terms of Gaussian Type Orbitals (GTOs). The basis sets for the molecular orbitals are normally adapted from standard quantum chemistry basis sets (obtainable, for example, from the NIST website (NIST 2001)). The basis set for the continuum orbitals is obtained by fitting numerical Bessel or Coulomb functions over a finite range (Nestmann and Peyerimhoff 1990) using the program GTOBAS (Faure et al. 2001). The 'continuum' GTOs are centred at the centre of mass of the system whereas the ones for the molecular orbitals are centred on the nuclei.

The most important element in the expansion (1) is the target wavefunctions $\phi_{i}$. As already mentioned, these wavefunctions are normally obtained using the configuration 
interaction method. The configurations included in the expansion are generated as products of molecular orbitals. How many and what sort of configurations depends mainly on the system studied. The hierarchy of quantum chemistry calculations for the electronic structure of small molecules is well established. A full CI can only be performed for few electron molecules, if a small basis set is chosen; a CASCI (complete active space configuration interaction: all excitations are performed among a set of orbitals that normally span the valence space of the target), CASS, CASSD or CASSDT (CAS plus single, double and triple excitations into a chosen set of virtual orbitals) are used for larger molecules. It has generally proven easier to maintain the balance between the $\mathrm{N}$-electron target representation and the $(\mathrm{N}+1)$-electron collision complex when a simple CAS model is used. In this model, $\mathbf{M}>\mathrm{N} / 2$ orbitals $\psi_{i}$ are selected; these normally comprise all those occupied in the main ground state configuration, plus a selection of orbitals that are presumed to be required for a good representation of the excited target states included in the expansion. If the orbitals $\psi_{i}$ are non-degenerate, each of them will generate two spin-orbitals $\bar{\psi}_{i}$; baring this in mind we can write:

$$
\left(\bar{\psi}_{1} \bar{\psi}_{2} \bar{\psi}_{3} \ldots \bar{\psi}_{M}\right)^{N}
$$

to indicate that the $\mathrm{N}$ electrons are distribute among all the $2 M$ spin-orbitals. It is understood that these orbitals will belong to different irreducible representations of the point group used in the calculation, and will be multiplied so that the configuration has the space and spin symmetry of the required target state. For target molecules with many electrons, it is customary to freeze the pairs of electrons that are most tightly bound (for example, those that correspond to closed inner shells or subshells). In this case the CAS is performed for less than the total $\mathrm{N}$ electrons, leaving the lowest energy orbitals doubly occupied in all configurations.

\subsection{Molecular R-matrix with pseudostates method}

The central idea of the RMPS method is the inclusion in the close-coupling expansion of a number of wavefunctions $\phi_{i}$ that represent pseudostates. These pseudostates are not true eigenstates of the target, but if chosen carefully, will represent a discretized version of the electronic continuum. Pseudostates are normally obtained by diagonalising the target electronic Hamiltonian expressed in an appropriate basis of configurations. When this is the case, three types of states are obtained:

(i) accurately represented bound states. These are states associated with eigenvalues that are close to the 'exact' energy of one of the target states.

(ii) pseudostates whose excitation energy lies below the ionisation threshold (I.T.). These states poorly represent bound states and their energy and character may not correspond to any true eigenstate of the system.

(iii) pseudostates associated to excitation energies lying above the I.T.. These states represent a discretized version of the target continuum.

The pseudostates with excitation energies above and below I.T., and in particular those close to it, can have a mixed bound/continuum character. Therefore, and although (in principle) transitions into states of type (iii) are often assumed to represent ionisation, it may 
be necessary to project out the bound component of these states (Kernoghan et al. 1995). This can only be done if a set of very accurate bound states can be generated, something that is far form easy for multielectronic systems, in particular molecules. In most of the atomic RMPS calculations reported to date, no projection method is used.

It is evident that for the $\phi_{i}$ to represent the continuum (albeit in a discretized fashion) some of the configurations included in the CI expansion must be able to represent the electronic density of an ionised state, that is, of the target with an extra positive charge and an electron that is no longer bound. This can be achieved by the appropriate choice of target orbitals and inclusion of specific configurations in the CI expansion, as will be explained in the next subsection.

2.2.1. Target states and pseudostates In order to generate configurations that describe an ionised target, we introduce in our calculations a new set of orbitals that we call pseudocontinuum orbitals (PCOs). These orbitals are used to describe the ionised electron. Hence, on top of the usual configurations (where all the electrons occupy molecular orbitals) another set in which one of the electrons occupies a PCO is included in the CI expansion. The following configurations are added to those generated by a standard CAS model (see equation 2):

$$
\left(\bar{\psi}_{1} \bar{\psi}_{2} \bar{\psi}_{3} \ldots \bar{\psi}_{\frac{N}{2}}\right)^{N-1} \lambda_{i}^{1} \quad i=1, \ldots ., I
$$

where $\lambda_{i}$ represents a PCO. Notice that only excitations from the main ground state configuration are allowed (this is why only N/2 orbitals are occupied by N-1 electrons). This choice of configurations does not allow for the representation of the excitation-ionisation process, but simplifies the determination of the pure ionisation cross section. Of course, other choices of configurations are possible and excitation-ionisation can also be studied, provided a clear distinction between ionisation and excitation-ionisation states can be made.

In our implementation, the PCOs are expanded in terms an even-tempered basis set (Schmidt and Ruedenberg 1979) of GTOs centred at the centre of mass of the system. In this type of basis sets, the exponents of the GTOs follow:

$$
\alpha_{i}=\alpha_{0} \beta^{(i-1)} \quad i=1, \ldots, L
$$

where by choosing different values of the parameters $\alpha_{0}$ and $\beta$ different basis sets can be systematically generated. This is very useful when an averaging procedure is used to eliminate pseudoresonances (see below). Additionally, even-tempered basis sets approach completeness as $\mathrm{L}$ approaches infinity, provided $\alpha_{0}$ and $\beta$ fulfil certain conditions (Schmidt and Ruedenberg 1979).

It is a necessary requirement for the R-matrix method to be valid that the electronic density of all the target states included in the close-coupling expansion (1) is contained inside the R-matrix box. This means, in practise, that the amplitudes of the basis functions used to expand the MOs must be negligible (normally $\leq 10^{-3}$ ) at the boundary. This must also hold for the GTOs expanding the PCOs thus imposing a lower limit on the values of $\alpha_{0}$ that can be employed. The choice of $\beta$ is dictated by two contradicting trends: smaller values provide a better distribution of pseudostates but make it more difficult to avoid linear dependence. 
The fundamental problem that arises when including pseudostates in the calculation is that of linear dependence amongst the basis functions. To avoid extra difficulties in the orthogonalisation, we ensure that:

$$
\alpha_{i}^{P C O s} \geq \alpha_{j}^{\text {continuum }} \quad \forall i, j
$$

This implies removing some GTOs from our standard continuum basis sets but does not undermine the representation of the scattered electron, as it is shown in the next section.

In the standard R-matrix polyatomic treatment (Morgan et al. 1998), the continuum orbitals are Schmidt orthogonalised to the already orthogonal target MOs. The resulting set of continuum orbitals are then made orthogonal using a symmetric orthogonalisation procedure. Several continuum orbitals may be deleted in this step; for this purpose, a deletion threshold, $\delta_{t h r s h}$, must be provided. For completeness, $\delta_{t h r s h}$ is usually kept at a low value unless linear dependence problems require it to be raised. In standard R-matrix calculations $\delta_{t h r s}$ varies with $a$ (and hence, with the continuum basis set) and is normally set to $\delta_{t h r s h}=10^{-7}$ for $a=10 a_{0}$. To allow for the inclusion of PCOs we implemented an extra orthogonalisation step: the PCOs are first Schmidt orthogonalised to the MOs and then symmetric orthogonalised among themselves (again, several PCOs may be deleted). The resulting set of MOs and PCOs is then treated as the MOs set in the standard calculation. We find that use of a bigger value of $\delta_{t h r s}$ is required both for PCOs and continuum orbitals in our MRMPS calculations.

2.2.2. Scattering Model Having represented, discretely, the bound and continuum states of the target, it is then necessary to construct the related target plus scattering electron (or $\mathrm{N}+1$ ) wavefunctions inside the R-matrix sphere. When the CI model chosen to describe the target states is that given by expressions (2) and (3), the following configurations are included in the $\mathrm{N}+1$ expansion:

$$
\begin{array}{ll}
\left(\bar{\psi}_{1} \bar{\psi}_{2} \bar{\psi}_{3} \ldots \bar{\psi}_{M}\right)^{N} \gamma_{j}^{1} & j=1, \ldots, J \\
\left(\bar{\psi}_{1} \bar{\psi}_{2} \bar{\psi}_{3} \ldots \bar{\psi}_{\frac{N}{2}}\right)^{N-1} \lambda_{i}^{1} \gamma_{j}^{1} & i=1, \ldots, I \quad j=1, \ldots, J
\end{array}
$$

where $\gamma_{j}$ represents a continuum orbital; and for the $L^{2}$ functions:

$$
\begin{aligned}
& \left(\bar{\psi}_{1} \bar{\psi}_{2} \bar{\psi}_{3} \ldots \bar{\psi}_{M}\right)^{N+1} \\
& \left(\bar{\psi}_{1} \bar{\psi}_{2} \bar{\psi}_{3} \ldots \bar{\psi}_{M}\right)^{N} \lambda_{i}^{1} \\
& \left(\bar{\psi}_{1} \bar{\psi}_{2} \bar{\psi}_{3} \ldots \bar{\psi}_{\frac{N}{2}}\right)^{N-1}\left(\lambda_{1} \ldots \lambda_{I}\right)^{2}
\end{aligned} \quad i=1, \ldots, I
$$

hence keeping a balance between the $\mathrm{N}$ and $\mathrm{N}+1$ calculation. As can be seen, some of the $L^{2}$ functions are such that 2 electrons (the ionised and the scattered one, one could say) occupy a PCO. Therefore, the PCO basis provides short range GTOs for the representation of the scattering electron. It could be argued that the second type of $L^{2}$ functions can supplement the correlation given by the target model. Nevertheless, tests showed that using only configurations of the type $\left(\bar{\psi}_{1} \bar{\psi}_{2} \bar{\psi}_{3} \ldots \bar{\psi}_{\frac{N}{2}}\right)^{N} \lambda_{i}^{1}$ undercorrelated the wavefunctions unbalancing the calculations.

The practical question that arises is how many and which states and pseudostates should be included in the calculation. The criterion we use is to include all the states obtained in 
our target calculation whose energy difference from the ground state is smaller than a certain value $\mathrm{E}_{\text {cut }}$. Here, we take $\mathrm{E}_{c u t}$ as being around 10-15 eV above the (vertical) I.T. of the target under study. Larger values of $\mathrm{E}_{c u t}$ are in principle possible but computationally too time consuming to be easily treated with the computers and program implementations currently available. Besides, at sufficiently high energies, other methods are equally accurate and more efficient.

An important consequence of the inclusion of pseudostates in the target expansion is the convergence of the polarizability. The slow convergence of these expansions is well known (Gil et al. 1994); so is the importance of accurately representing the polarizability of the target for low energy collisions. Tests for $\mathrm{H}_{3}^{+}$and $\mathrm{H}_{2}$ showed that increasing the number of target states in the expansion without the use of pseudostates does not lead to a converged polarizability. Inclusion of a discretised representation of the target continuum is clearly essential. We also use the converge of the polarizability as a test to ensure that an adequate number of pseudostates has been included in the calculation.

2.2.3. K-matrix and cross section calculation The collision information (eigenphase sums, resonance parameters, cross sections, etc..) is determined by propagating the R-matrix from the boundary between the inner and outer region to an asymptotic distance where the form of the radial part of the wavefunction describing the scattering electron is well known. In the outer region, each of the target states generates a number of channels that together describe all the possible outcomes of the collision. The more target states that are included in the calculation, the larger the number of channels in the outer region. In a standard R-matrix calculation, the diagonalisation of the $\mathrm{N}+1$ Hamiltonian to determine the wavefunctions of equation (1) is computationally the most demanding step. But for the MRMPS, the number of channels greatly increases, and it is the propagation step that takes the longest therefore restricting the number of target bound and pseudostates that can be used in the close-coupling expansion.

The inclusion of pseudostates in a calculation introduces unphysical, spurious resonances above the ionisation threshold known as pseudoresonances. For cationic targets each pseudostate supports a series of Feshbach type pseudoresonances associated with the Rydberg series converging to them. Below the I.T. these (pseudo)resonances give an approximate representation of real resonance series of the system. Several methods have been proposed to deal with above I.T. pseudoresonances in electron-atom collisions. In atomic RMPS calculations, they are eliminated by performing a weighted average of several calculations with different basis for the PCOs (Bartschat and Bray 1996). This method was not effective in the case of $\mathrm{H}_{3}^{+}$, due to the large number of pseudoresonances (see section 3). We found that a convolution procedure similar to the one proposed by Meyer et al. (1995) followed by an averaging of the convoluted results, is the best suited to deal with the problem in the ionisation cross section. A Gaussian function of variable width was used to convolute each cross section 
(for each PCO basis) and the results were then averaged to produce our final cross section:

$$
\sigma^{I O N}(E)=\frac{1}{4 \sqrt{\frac{\pi}{\sqrt{E}}}} \sum_{i}^{4} \int_{E_{m}}^{E_{M}} e^{-\sqrt{E}\left(E^{\prime}-E\right)^{2}} \sigma_{i}^{i o n}\left(E^{\prime} ; \alpha_{0 i}\right) \mathrm{d} E^{\prime}
$$

Here, $E_{m}$ is taken to be $0.5 \mathrm{E}_{h}$ smaller than the I.T. and $E_{M}$ is at least $0.5 \mathrm{E}_{h}$ bigger than the maximum energy for which $\sigma^{I O N}$ is presented. This method eliminates all resonances, including physical ones; a different technique should be used if these resonances need to be preserved. Because virtually no pseudoresonances where present in the $\mathrm{H}_{2}$ ionisation cross section, the simple averaging procedure was sufficient to produce a satisfactory final result.

\section{Calculations on $\mathbf{H}_{3}^{+}$}

As mentioned in the introduction, we chose to first apply the method to $\mathrm{H}_{3}^{+}$, the smallest polyatomic ion. Being positively charged, its electronic states are fairly compact and that ensures that we can use the standard $a=10 \mathrm{a}_{0}$ radius for the R-matrix box. By keeping $a$ small, we keep the calculation within the bounds of what we can treat. The larger $a$, the larger the number of continuum basis functions necessary for correctly representing the scattering electron and hence the larger the $\mathrm{CI}$ performed to obtain the $\mathrm{N}+1$ wavefunctions. Besides, our tests show that as $a$ increases the single centre GTO expansion becomes increasingly poor at representing the (oscillating) continuum near the R-matrix boundary. It is unlikely that we would be able to use $a \geq 15 a_{0}$ with the current implementation of the method.

Earlier theoretical work on electronically inelastic processes (Orel 1992, Faure and Tennyson 2002b) provided cross sections for electronic excitation for energies up to $20 \mathrm{eV}$ and widths and positions for the lowest-lying resonances. Both these calculations include 6 bound electronic target states in the close-coupling expansion and the agreement between them is fairly good.

From a full CI calculation we determined the vertical I.T. of $\mathrm{H}_{3}^{+}$to be $33.47 \mathrm{eV}$. We have calculated cross sections for electronic excitation both above and below the I.T. and ionisation cross sections up to $43 \mathrm{eV}$. The characteristics of our calculation are the following: for the MOs, we followed Faure and Tennyson (2002b) and used the basis set from Orel (1992) removing from it the two GTOs with the smallest exponents. In contrast to previous studies, we built MOs corresponding to $\mathrm{H}_{3}^{2+}$. In this way we tried to ensure that configurations with single excitations into PCOs represented an electronic distribution similar to that of an ionised state of $\mathrm{H}_{3}^{+}$.

Several PCO bases were tested: we needed to avoid linear dependence problems and, as discussed below, we required a fairly homogeneous distribution of pseudostates. The first bases tested (with $\beta=1.5$ and $\alpha_{0}=0.14,0.15,0.16,0.17$ ) had few pseudostates associated with the open channels in the energy range 33-43 eV. Furthermore, the first pseudostate corresponding to a continuum state was $2 \mathrm{eV}$ above the I.T.. These calculations resulted in cross sections that showed a 'step' behaviour and displayed a threshold for ionisation that was $2 \mathrm{eV}$ above the true threshold. Reducing $\beta$ was shown to improve the pseudostate distribution, also reducing the gap between the first continuum state and the I.T.. The best calculation was 
performed using $\beta=1.3$, since $\beta=1.2$ caused linear dependence problems. The same set of $\alpha_{0}$ mentioned before, $\alpha_{0}=0.14,0.15,0.16,0.17$, was used. The minimum value for $\alpha_{0}$ was selected to ensure that the target states did not leak outside the R-matrix box. In all cases we restricted the PCO basis to $l \leq 2$ due to computational limitations.

The calculations were performed using the $\mathrm{C}_{2 v}$ point group $\left(\mathrm{H}_{3}^{+}\right.$in its equilibrium geometry belongs to the $\mathrm{D}_{3 h}$ point group). Using the notation for the irreducible representations of $\mathrm{C}_{2 v}$, the $\mathrm{CI}$ model can be expressed in the following way:

$$
\begin{aligned}
& \left(1-5 a_{1} 1-3 b_{1} 1-3 b_{2} 1 a_{2}\right)^{2} \\
& 1 a_{1}^{1}\left(6-35 a_{1} 4-17 b_{1} 4-18 b_{2} 2-8 a_{2}\right)^{1}
\end{aligned}
$$

where the orbitals in the first line are MOs and the ones between brackets in the second line are PCOs. The excitation thresholds are in reasonable good agreement with previous calculations, as shown in Table 1. In figure 1 we have plotted the energy distribution of (bound and pseudo) states obtained with different $\left(\alpha_{0}, \beta\right)$ values. As can be seen, the lowest bound states have almost the same energy. As the energy increases, the changes in the distribution of states (with changing $\alpha_{0}$ ) become more significant. All the distributions for $\beta=1.3$ show the desirable property of having one or several states just above the I.T.. This is in contrast to the state distribution obtained with $\beta=1.5$, also shown in the figure for $\alpha_{0}=0.14$.

For the continuum we adapted the basis set from Faure and Tennyson (2002b). In order to ensure that condition (5) was fulfilled, the largest exponents were moved from the continuum to the PCO basis. The third type of configurations from (7) compensates for this thus maintaining the quality of the continuum representation. We find that use of $\delta_{\text {thrsh }} \geq 2 \times 10^{-6}$ was required to avoid linear dependence both for PCOs and continuum orbitals. The closecoupling expansion included 64 target bound and continuum states, which corresponds to using at least $E_{\text {cut }}=45 \mathrm{eV}$. We chose to keep the number of states of each symmetry included in the close-coupling constant: as the distribution of states is different for each PCO basis, different $E_{c u t}$ values are then implied for each PCO basis. Using 64 states also ensures that the polarizability has converged (see Table 1 in Gorfinkiel and Tennyson (2004)). Our tests showed that $\alpha_{\perp}$ does not converge unless pseudostates, that is a discretised representation of the target continuum, are included in the expansion. Inclusion of 64 target states brings both components of the polarizability to within $2 \%$ of the high accuracy result.

Our low energy eigenphase sums are in general agreement with previous 6 state calculations (Orel and Kulander 1993, Faure and Tennyson 2002b). However, the positions of the low-lying Feshbach resonances are all shifted, mostly downward. Resonance positions depend on threshold positions, so a more consistent comparison is that of the quantum defect parameters, presented in Table 2. Since the Faure and Tennyson (2002b) calculations was performed, a bug affecting the treatment of degenerate states has been corrected in the Rmatrix suite. For this reason, we re-ran the 6 state calculation with $5 \mathrm{a}_{1}, 3 \mathrm{~b}_{1}, 3 \mathrm{~b}_{2}$ and $1 \mathrm{a}_{2}$ MOs corresponding to $\mathrm{H}_{2}^{+}$. As can be seen, the $\alpha$ parameters of the calculation that includes pseudostates are about 0.05/0.06 bigger that those of our 6 state calculation. This result is probably a consequence of our correct representation of the polarizability and could be significant for processes such as ion-pair formation in the dissociative recombination of 
$\mathrm{H}_{3}^{+}$(Kalhori et al. 2004).

Figure 2 shows our cross section for excitation into the two lowest electronically excited state. These are the ${ }^{3} \mathrm{E}$ ' and ${ }^{1} \mathrm{E}$ ' that in the $\mathrm{C}_{2 v}$ point group each splits into $\mathrm{A}_{1}$ and $\mathrm{B}_{2}$. The four $\beta=1.3$ bases for the PCOs give almost identical results below the I.T. so only one is shown in the figure. Above the I.T., the position of the pseudoresonances is different for each basis. The averaging plus convolution procedure used for the ionisation cross section did not produce a smooth result for the above I.T. cross section. Large bumps were still visible where bunches of resonances converge to a pseudothreshold in the non-averaged results. This is probably a sign that the contribution of the resonant process is more significant for electronic excitation than ionisation. Hence, a cubic fit to the average of the four $\beta=1.3$ pseudostate calculations is shown in the figures as our recommended value in this energy region.

The ionisation cross section is shown in figure 3. Several results have been plotted here: figure $3 \mathrm{a}$ shows the ionisation cross section for each PCO basis with $\beta=1.3\left(\sigma_{i}^{\text {ion }}\left(E ; \alpha_{0 i}\right)\right.$ in equation 8). It is noticeable that the pseudoresonances are in different positions for the different PCO basis. Also plotted in figure $3 \mathrm{a}$ is $\sigma_{i}^{i o n}(E ; 0.14)$ for $\beta=1.5$. The nonphysical step behaviour of this cross section, caused by the unsatisfactory distribution of the pseudostates, is clearly visible. Figure $3 \mathrm{~b}$ shows the average of the $\beta=1.3 \sigma_{i}^{\text {ion }}\left(E ; \alpha_{0 i}\right)$ showing that the pseudoresonances do not disappear when the average is performed. When the convolution plus averaging procedure of equation 8 is applied, we obtain the $\sigma^{I O N}$ plotted in figure 3c. The pseudoresonances have disappeared in this result, but some mild non-physical oscillations persist; we consider these to be within the error of our calculations. Finally, also plotted is a low energy fit following the Wannier threshold law (Wannier 1953) that

gives an exponent of 1.05589 for this process. The agreement with our non-convoluted cross section is reasonable. However, the convolution process modifies the very near-threshold behaviour: this is just a numerical problem, related to the width of the Gaussian included in eq. (8). Hence, this method should not be used to provide results for I.T. $<\mathrm{E}<\mathrm{I}$.T. $+\Gamma$, where $\Gamma=0.5 \mathrm{eV}$ for $\mathrm{H}_{3}^{+}$but may vary with the target.

\section{Calculations on $\mathrm{H}_{2}$}

For a benchmark calculation, $\mathrm{H}_{2}$ is, in principle, the best choice. Nevertheless, the diffuse character of its low-lying excited states means that an R-matrix radius $a=20 \mathrm{a}_{0}$ (Branchett and Tennyson 1990) is needed if one is to include several of these states in the calculation. This being beyond our means and considering that our interest was to calculate ionisation cross sections, we chose a simpler model. As a first approximation to the problem, only the ground and first excited states were represented accurately. The remaining states are (bound and continuum) pseudostates. Within this model, we are able to set $a=10 \mathrm{a}_{0}$.

We used a fairly small 6-31G** basis set (Hariharan and Pople 1973) and built $\mathrm{H}_{2}^{+}$MOs. For the PCOs it was again necessary to test different basis sets. We started with the most diffuse PCO basis used for $\mathrm{H}_{3}^{+}$, that with $\beta=1.3$ and $\alpha_{0}=0.14$, but found it to give linear dependence. After various test calculations, we found that $\beta=1.4$ and $\alpha_{0}=0.17,0.18,0.19$ gave the right pseudostate distribution while avoiding linear dependence provided $\delta_{\text {thr } h}=2 \times 10^{-4}$, 
much bigger than for $\mathrm{H}_{3}^{+}$, was used. For the orthogonalization of the continuum GTOs, the same $\delta_{\text {thrsh }}=2 \times 10^{-6}$ was sufficient. The point group $\mathrm{D}_{2 h}$ was used and CI model was the following:

$$
\begin{aligned}
& \left(1-3 \mathrm{a}_{g} 1 \mathrm{~b}_{2 u} 1 \mathrm{~b}_{3 u} 1-2 \mathrm{~b}_{1 u} 1 \mathrm{~b}_{2 g} 1 \mathrm{~b}_{3 g}\right)^{2} \\
& 1 \mathrm{a}_{g}^{1}\left(4-20 \mathrm{a}_{g} 2-8 \mathrm{~b}_{2 u} 2-8 \mathrm{~b}_{3 u} 1-5 \mathrm{~b}_{1 g} 3-9 \mathrm{~b}_{1 u} 2-6 \mathrm{~b}_{2 g} 2-6 \mathrm{~b}_{3 g}\right)^{1}
\end{aligned}
$$

where again the orbitals in the first line are MOs and the ones between brackets in the second line are the PCOs. The distribution of states can be seen in figure 4. Again, the larger differences between different PCO bases occur for the pseudostates above the I.T.. The ground state energy for all $\alpha_{0}$ was $-1.165 \mathrm{E}_{h}$, compared to the very accurate value of $-1.1744 \mathrm{E}_{h}$ from Kolos and Szalewicz (1986). The threshold for vertical excitation into the $\mathrm{b}^{3} \Sigma_{u}^{+}$, the lowest excited state, is $10.45 \mathrm{eV}$ again in excellent agreement with the accurate value of $10.62 \mathrm{eV}$ provided by Kolos and Wolniewicz (1965).

The continuum basis set was the standard for neutral targets from Faure et al. (2001) and again some of the highest exponents were moved to the PCO basis. Choosing an $\mathrm{E}_{c u t} \geq$ $35.5 \mathrm{eV}, 30$ states were included in the close-coupling expansion. The polarizability obtained with these states is fairly good, each of the components being within $5 \%$ of the accurate value (see Table 3).

The cross section for excitation into $\mathrm{b}^{3} \Sigma_{u}^{+}$, the first excited state of $\mathrm{H}_{2}$, is plotted in figure 5. The three PCO bases give very similar results, and show a peak around 13.7$13.9 \mathrm{eV}$ that corresponds to a ${ }^{2} \Sigma_{g}^{+}$and a ${ }^{2} \Sigma_{u}^{+}$resonance which are very close together. The parent states of these Feshbach resonances are poorly represented in our calculation, their thresholds being at least $1.5 \mathrm{eV}$ higher than those of Trevisan and Tennyson (2002). Hence, the resonances appearance at much higher energies than in their calculation. (The calculation of Trevisan and Tennyson (2002) is similar to that of Branchett and Tennyson (1990), but with consistent phases (Tennyson 1997) in the $\mathrm{N}$ and $\mathrm{N}+1$ calculation). None of the other previous calculations (Lima et al. 1985, Baluja et al. 1985, Lima et al. 1988) show a resonance structure, and the few experimental points available (Hall and Andric 1984, Nishimura and Danjo 1986, Khakoo et al. 1987, Khakoo and Segura 1994) are insufficient to describe any resonances. Our biggest calculation, that includes 41 states using $\alpha_{0}=0.17$, shows a reasonable agreement with experiment at $40 \mathrm{eV}$; it also shows that the 30 state results are converged for $\mathrm{E}<30 \mathrm{eV}$.

In figure 6 we have plotted the ionisation cross sections for the three different PCOs. The first significant difference with $\mathrm{H}_{3}^{+}$is the absence of visible pseudoresonances: one can just about see a few spikes that are probably extremely narrow resonances. This means that in this case, a simple averaging of the cross sections provides our 'recommended value' result, also plotted in the figure. The agreement with the experimental data (Krishnakumar and Srivastava 1994, Straub et al. 1996) and the cross section obtained by the Binary Encounter Bethe method (BEB, Kim and Rudd 1994) is extremely good, even up to almost $15 \mathrm{eV}$ above the I.T..

In our calculations, we have used the adiabatic I.T., $15.4 \mathrm{eV}$, as the cut-off value to distinguish between bound and continuum states. This choice, albeit somewhat inconsistent 
with the fact that our calculations are performed in the FN approximation, is necessary to get the correct threshold. There is a gap (see figure 4) of around $2 \mathrm{eV}$ in the pseudostate distribution just above the vertical I.T., $16.4 \mathrm{eV}$, that produces a non-physical cross section if that threshold is used. Unfortunately, none of the PCO basis tested gave a better pseudostate distribution, partly due to do the fact that linear dependence problems are more severe than for $\mathrm{H}_{3}^{+}$. It is possible that the pseudostates lying just below the vertical I.T. have a large continuum character. In this case, a projection method would produce a non-zero ionisation cross section even below the vertical threshold. In any case, to obtain cross sections showing the adiabatic I.T. one would need to include the nuclear motion in the calculation, something achievable for a diatomic like $\mathrm{H}_{2}$.

\section{Conclusions}

The MRMPS method has been used to calculate ionisation cross sections as well as electronic excitation cross sections above the ionisation threshold. Results for $\mathrm{H}_{2}$ show excellent agreement between experimental data and the MRMPS calculations, confirming its validity. The difficulty in using the MRMPS method lies in the correct choice of $\alpha_{0}$ and $\beta$ for the PCO basis and the corresponding $\delta_{t h r s h}$. More systems need to be studied to establish whether it is possible to define some rules or systematic strategy for the choice of these parameters. At the moment, a trial and error procedure must be used to test for the presence of linear dependence in the calculations and to obtain a good distribution of pseudostates. Similarly, some further developments would still be desirable. The difficulties with the pseudostate distribution for $\mathrm{H}_{2}$ point to the need for a procedure to project out the bound component of the pseudostates that lie very close to the ionisation threshold. Likewise, a method to top-up the partial wave expansion will certainly be needed if higher energies are to be explored. For example, an approach similar to that used in RMPS calculations (Bartschat and Bray 1996b) where a noexchange approximation is used for hight angular momentum, could be implemented.

The MRMPS method could also prove very useful for determining cross sections below the ionisation threshold. Inclusion of pseudostates has been shown to significantly improve the convergence of the polarizability for both systems studied. This has an effect on the resonance parameters (very relevant for dissociation processes) as well as the excitation cross sections. Finally, the method is fully general and has been implemented as part of the UK polyatomic R-matrix code. We believe that it will enable the study of electron collisions with molecular anions as well as excitation to high lying electronic states.

\section{Acknowledgments}

This work was supported by the UK Engineering and Physical Sciences Research Council. Calculations were carried out on the Ra Supercomputer, at the HiPerSPACE Computing Centre, UCL. We are indebted to Alex Faure for his advice on the $\mathrm{H}_{3}^{+}$calculations and Natalia Vinci for help with the R-matrix codes. We thank Klaus Bartschat and Phil Burke for helpful discussions. 


\section{References}

Augspurger J D and Dykstra C E 1998 J. Chem. Phys. 88, 3817.

Baluja K L, Noble C J and Tennyson J 1985 J. Phys. B: At. Mol. Phys. 18, L851.

Bartschat K and Bray I 1996 Phys. Rev. A 54, R1002.

Bartschat K, Hudson E T, Scott M P, Burke P G and Burke V M 1996 J. Phys. B: At. Mol. Phys. 29, 115.

Branchett S E and Tennyson J 1990 Phys. Rev. Lett. 64, 2889.

Bray I, Fursa D V, Kheifets A S and Stelvobics A T 2002 J. Phys. B: At. Mol. Phys. 35, R117.

Brunger M J and Buckman S J 2002 Physcis Reports 357, 215.

Burke P G, Noble C J and Scott M P 1987 Proc. R. Soc. A 410, 911.

Deutsch H, Becker K, Matt S and Märk T D 2000 Int. J. Mass Spec. 197, 37.

Faure A, Gorfinkiel J D, Morgan L A and Tennyson J 2001 Computer Phys. Commun. p. 224.

Faure A and Tennyson J 2002a J. Phys. B: At. Mol. Phys. 35, 3945.

Faure A and Tennyson J 2002b J. Phys. B: At. Mol. Phys. 35, 1865.

Ghazaly $\mathrm{M} \mathrm{E}$ and et al in preparation.

Gil T J, Lengsfield B H, McCurdy C W and Rescigno T N 1994 Phys. Rev. A 49, 2551.

Gorfinkiel J D and Tennyson J 2004 J. Phys. B: At. Mol. Phys. 37, L343.

Hall R I and Andric L 1984 J. Phys. B: At. Mol. Phys. 17, 3815.

Hariharan P and Pople J 1973 Theoret. Chimica Acta 28, 213.

Huo W M 2001 Phys. Rev. A 64, 042719.

Huo W M and Gianturco F A, eds 1995 Computational Methods for Electron-Molecule Collisions Plenum Press.

Kalhori S, Thomas R, Al-Khalili A, Ehlerding A, Hellberg F, Neau A, Larsson M, Larson Å, Huneycutt A J, McCall B J, Djurić N, Dunn G H, Semaniak J, Novotny O, Paál A, Österdahl F and Orel A E 2004 Phys. Rev. A 69, 022713.

Kernoghan A A, McAlinden M T and Walters H R J 1995 J. Phys. B: At. Mol. Phys. 28, 1079.

Khakoo M A and Segura J 1994 J. Phys. B: At. Mol. Phys. 27, 2355.

Khakoo M A, Trajmar S, McAdams R and Shyn T 1987 Phys. Rev. A 35, 2832.

Kim Y K and Rudd M E 1994 Phys. Rev. A 50, 3954.

Kokoouline V and Greene C H 2003 Phys. Rev. Lett. 90, 133201.

Kolos W and Szalewicz K 1986 J. Chem. Phys. 84, 3278.

Kolos W and Wolniewicz L 1965 J. Chem. Phys. 43, 2429.

Krishnakumar E and Srivastava S K 1994 J. Phys. B: At. Mol. Phys. 27, L251.

Lima M A P, Gibson T L, Huo W M and McKoy V 1985 J. Phys. B: At. Mol. Phys. 18, L865-870.

Lima M A P, Gibson T L, McKoy V and Huo W M 1988 Phys. Rev. A 38, 4527.

McCall B J, Geballe T, Hinkle K H and Oka T 1998 Science 279, 1910.

McCall B J, Huneycutt A, Saykally R, Geballe T, Djurić N, Dunn G, Semaniak J, Novotny O, Al-Khalili A, Ehlerding A, Hellberg F, Kalhori S, Neau A, Thomas R, Sterdahl F and Larsson M 2003 Nature 422, 500.

Meyer K W, Greene C H and Bray I 1995 Phys. Rev. A 52, 1334.

Morgan L A, Gillan C J, Tennyson J and Chen X 1997 J. Phys. B: At. Mol. Phys. 30, 4087.

Morgan L A, Tennyson J and Gillan C J 1998 Computer Phys. Commun. 114, 120.

Nestmann B M and Peyerimhoff S D 1990 J. Phys. B: At. Mol. Phys. 23, L773.

Nishimura H and Danjo A 1986 J. Phys. Soc. Jpn. 55, 1986.

NIST 2001 'Nist, standard reference database 69 - july 2001 release: Nist chemistry webbook,' webbook.nist.gov/chemistry.

Orel A E 1992 Phys. Rev. A 46, 1333.

Orel A E and Kulander K C 1993 Phys. Rev. Lett. 71, 4315.

Pindzola M S and Robicheaux F J 2000 Phys. Rev. A 61, 052707.

Rescigno T N, Baertschy M, Isaacs W A and McCurdy C W 1999 Science 286, 2474.

Schmidt M W and Ruedenberg K 1979 J. Chem. Phys. 71, 3951.

Straub H C, Renault P, Lindsay B G, Smith K A and Stebbings R F 1996 Phys. Rev. A 54, 2146.

Tennyson J 1996 J. Phys. B: At. Mol. Phys. 29, 6185. 
Tennyson J 1997 Computer Phys. Commun. 100, 26.

Tennyson J and Morgan L A 1999 Phil. Trans. A 357, 1161.

Trevisan C S and Tennyson J 2002 Private communication. Unpublished.

Wannier G H 1953 Physical Review 90, 817.

Bartschat K and Bray I 1996 J. Phys. B: At. Mol. Phys. A 29, L577. 


\section{Tables and table captions}

Table 1. Ground state (in $\mathrm{E}_{h}$ ) and vertical excitation energies for $\mathrm{H}_{3}^{+}$(in eV) for (a) the PCOs basis with $\alpha_{0}=0.14$ and $\beta=1.3$, (b) 6 state calculation without pseudostates (b) and (c) Orel (1992).

\begin{tabular}{lllccc}
\hline \multicolumn{2}{c}{ Electronic state } & & \multicolumn{3}{c}{ Vertical excitation } \\
\cline { 1 - 2 } \cline { 5 - 6 } $\mathrm{D}_{3 h}$ & $\mathrm{C}_{2 v}$ & & $\mathrm{a}$ & $\mathrm{b}$ & $\mathrm{c}$ \\
\hline$X^{1} \mathrm{~A}_{1}$ & ${ }^{1} \mathrm{~A}_{1}$ & & -1.3242 & -1.3049 & - \\
${ }^{3} \mathrm{E}^{\prime}$ & ${ }^{3} \mathrm{~A}_{1}+{ }^{3} \mathrm{~B}_{2}$ & & 14.65 & 14.16 & 14.81 \\
${ }^{1} \mathrm{E}$, & ${ }^{1} \mathrm{~A}_{1}+{ }^{1} \mathrm{~B}_{2}$ & & 19.35 & 19.04 & 19.61 \\
${ }^{3} \mathrm{~A}^{\prime}{ }_{2}$ & ${ }^{3} \mathrm{~B}_{1}$ & & 20.41 & 19.99 & 20.73 \\
\hline
\end{tabular}

Table 2. Complex quantum defect parameters of the lowest nine resonances of $\mathrm{H}_{3}^{+}$at the equilibrium geometry. Column A corresponds to a 64 state calculation; column B corresponds to a 6 states calculation with no pseudostates. Powers of ten are given in parentheses.

\begin{tabular}{llcccc}
\hline \multicolumn{2}{c}{ Symmetry } & \multicolumn{2}{c}{ This work $\mathrm{A}$} & \multicolumn{2}{c}{ This work B } \\
\cline { 1 - 5 } $\mathrm{D}_{3 h}$ & $\mathrm{C}_{2 v}$ & $\mathrm{n}-\alpha$ & $\beta$ & $\mathrm{n}-\alpha$ & $\beta$ \\
\hline${ }^{2} \mathrm{E}^{\prime}$ & ${ }^{2} \mathrm{~A}_{1}+{ }^{2} \mathrm{~B}_{2}$ & 1.52 & 0.037 & 1.57 & 0.041 \\
${ }^{2} \mathrm{~A}_{1}^{\prime}$ & ${ }^{2} \mathrm{~A}_{1}$ & 1.64 & 0.015 & 1.72 & 0.016 \\
${ }^{2} \mathrm{~A}_{2}$ & ${ }^{2} \mathrm{~B}_{2}$ & 1.88 & $7.9(-5)$ & 1.96 & $6.7(-5)$ \\
${ }^{2} \mathrm{E}^{\prime}$ & ${ }^{2} \mathrm{~B}_{1}+{ }^{2} \mathrm{~A}_{2}$ & 1.89 & 0.012 & 1.96 & 0.012 \\
${ }^{2} \mathrm{E}^{\prime}$ & ${ }^{2} \mathrm{~A}_{1}+{ }^{2} \mathrm{~B}_{2}$ & 1.92 & 0.013 & 1.96 & 0.016 \\
${ }^{2} \mathrm{E}^{\prime}$ & ${ }^{2} \mathrm{~A}_{1}+{ }^{2} \mathrm{~B}_{2}$ & 2.67 & 0.028 & 2.72 & 0.026 \\
${ }^{2} \mathrm{~A}_{1}^{\prime}$ & ${ }^{2} \mathrm{~A}_{1}$ & 2.76 & 0.010 & 2.82 & $9.3(-2)$ \\
${ }^{2} \mathrm{E}^{\prime}$ & ${ }^{2} \mathrm{~A}_{1}+{ }^{2} \mathrm{~B}_{2}$ & 2.87 & $5.3(-3)$ & 2.92 & $5.0(-3)$ \\
${ }^{2} \mathrm{E}^{\prime}$, & ${ }^{2} \mathrm{~B}_{1}+{ }^{2} \mathrm{~A}_{2}$ & 2.91 & 0.012 & 2.96 & 0.012 \\
${ }^{2} \mathrm{~A}_{1}^{\prime}$ & ${ }^{2} \mathrm{~A}_{1}$ & - & - & 2.97 & $7.7(-2)$ \\
\hline
\end{tabular}


Table 3. Polarizabilities of $\mathrm{H}_{2}$ using a PCO basis with $\beta=1.4$ and $\alpha_{0}=0.17$. Seven state result from Branchett and Tennyson (1990). Accurate ab initio value from Augspurger and Dykstra (1998).

\begin{tabular}{lcc}
\hline States in close-coupling expansion & $\alpha_{\|}$ & $\alpha_{\perp}$ \\
\hline 7 (bound states) & -5.08 & 0.00 \\
30 (states up to $\left.\mathrm{E}_{c u t}=35.5 \mathrm{eV}\right)$ & -6.117 & -4.488 \\
$66\left(\right.$ states up to $\left.\mathrm{E}_{c u t}=38 \mathrm{eV}\right)$ & -6.144 & -4.543 \\
$120(30$ for each symmetry) & -6.145 & -4.544 \\
Accurate $a b$ initio value & -6.445 & -4.507 \\
\hline
\end{tabular}




\section{Figure captions}

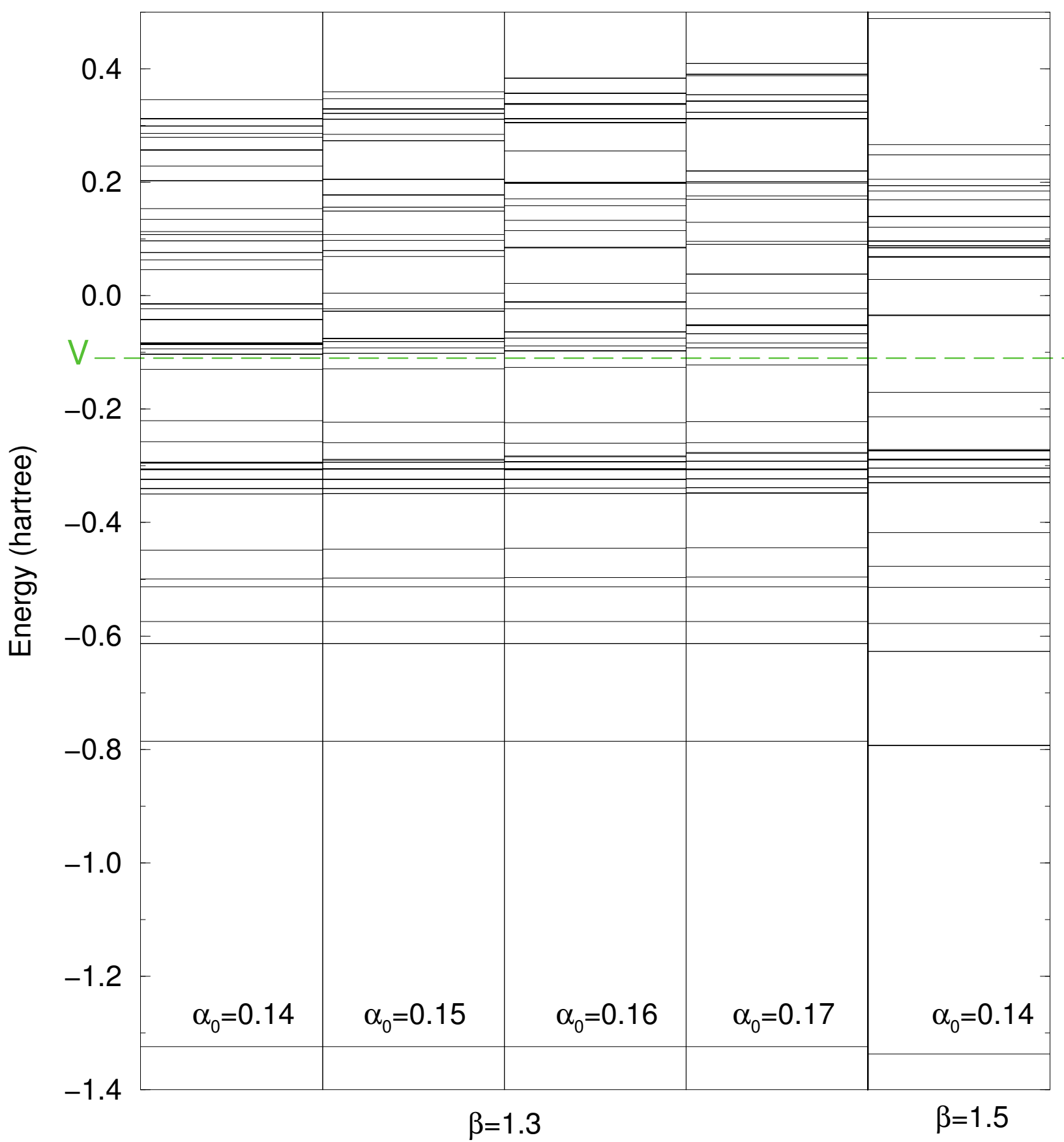

Figure 1. Target state distribution for $\mathrm{H}_{3}^{+}$for $\delta_{t h r s h}=5 \times 10^{-6}$ and the $\left(\alpha_{0}, \beta\right)$ values indicated in the figure. The dashed line indicated with $\mathrm{V}$ corresponds to the vertical ionisation threshold. 


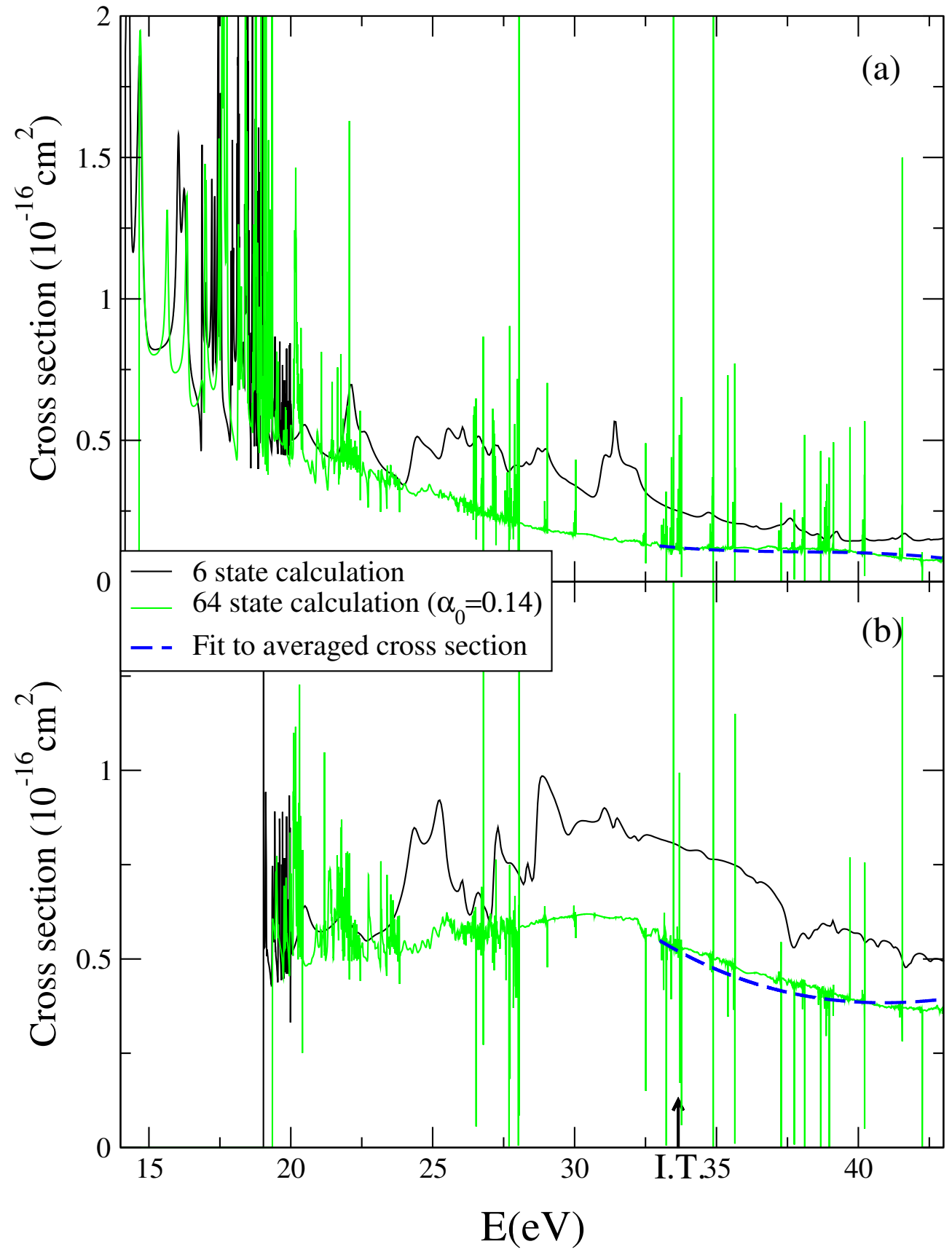

Figure 2. Integral cross section for excitation into: (a) first excited state $\left({ }^{3} \mathrm{E}^{\prime}\right)$ and (b) second excited state $\left({ }^{1} \mathrm{E}^{\prime}\right)$ of $\mathrm{H}_{3}^{+}$. The arrow indicates the ionisation threshold (I.T.). 

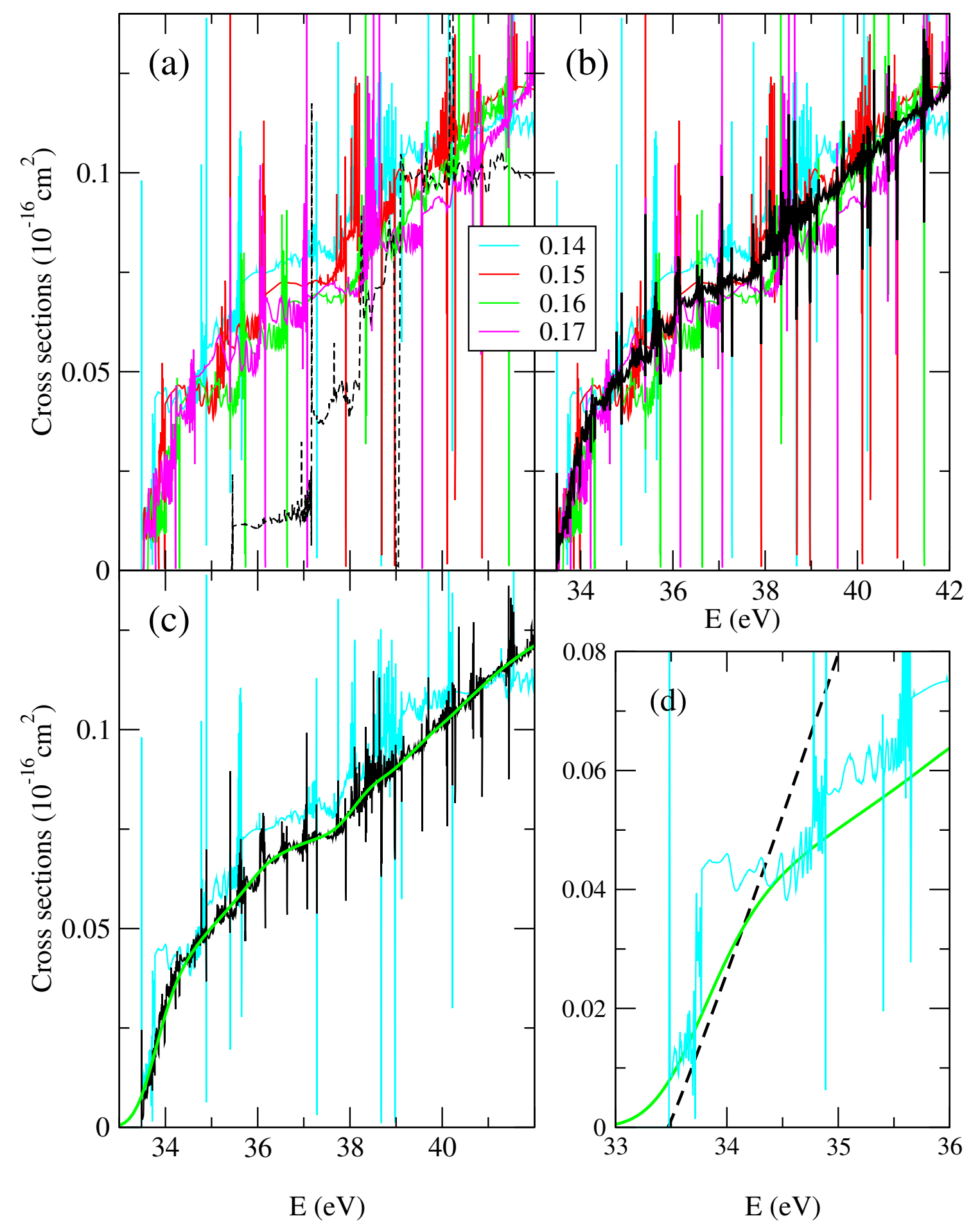

Figure 3. Total ionisation cross section for $\mathrm{H}_{3}^{+}$. Panels (a) and (b) show results for four different PCO basis with $\beta=1.3$ and the $\alpha_{0}$ values indicated in the graph. In (a) dashed line correspond to PCO basis set with $\alpha_{0}=0.14$ and $\beta=1.5$. In (b) the dark full line corresponds to the averaged cross section also plotted in (c). In (c) and (d) thick smooth full line is an average of the four $\beta=1.3$ convoluted cross sections and the light full line corresponds to $\alpha_{0}=0.14$. Thick dashed line in (d) is a low energy fit following the Wannier threshold law: $\sigma \propto \mathrm{E}^{1.05589}$ (Wannier 1953). 


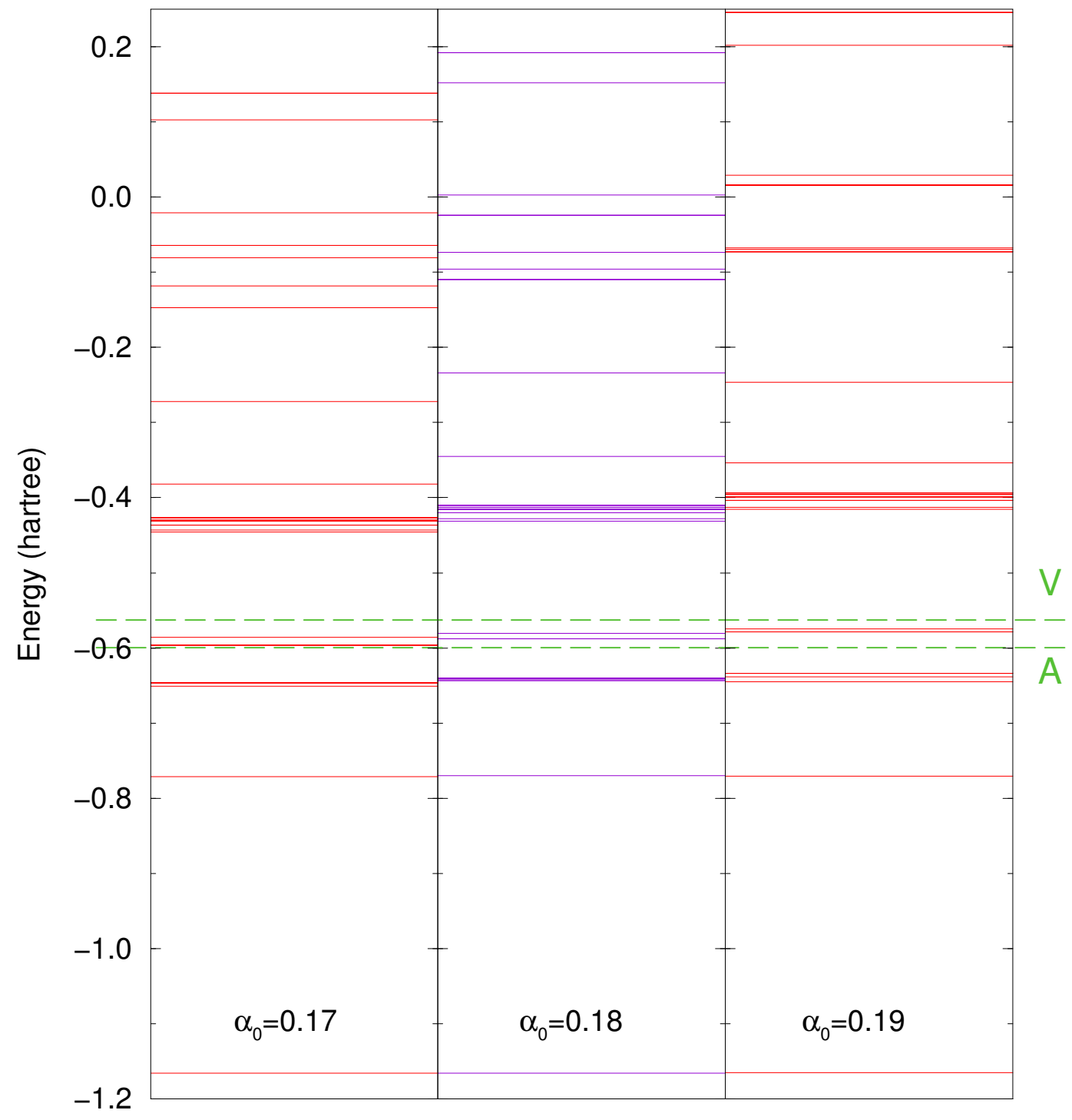

Figure 4. Target state distribution for $\mathrm{H}_{2}$ for $\delta_{t h r s h}=2 \times 10^{-4}, \beta=1.4$ and the $\alpha_{0}$ indicated in the figure. The dashed lines correspond to the vertical $(\mathrm{V})$ and adiabatic $(\mathrm{A})$ ionisation thresholds. 


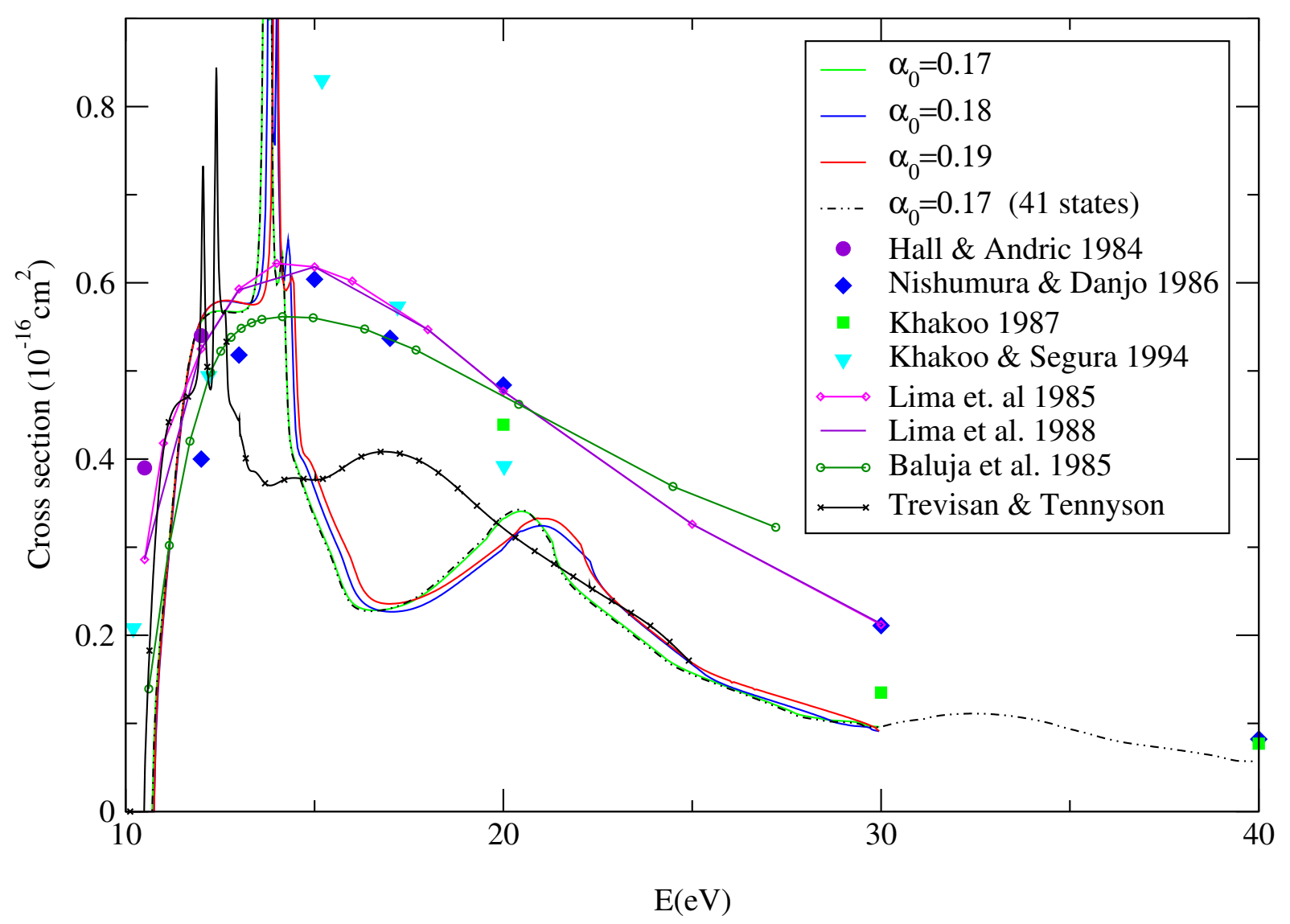

Figure 5. Integral cross section for excitation into the first excited state of $\mathrm{H}_{2}, \mathrm{~b}^{3} \Sigma_{u}^{+}$, for $\beta=1.4$ and the $\alpha_{0}$ indicated in the figure. Previous theoretical and experimental results as indicated in figure. Trevisan and Tennyson (2002) are unpublished results. 


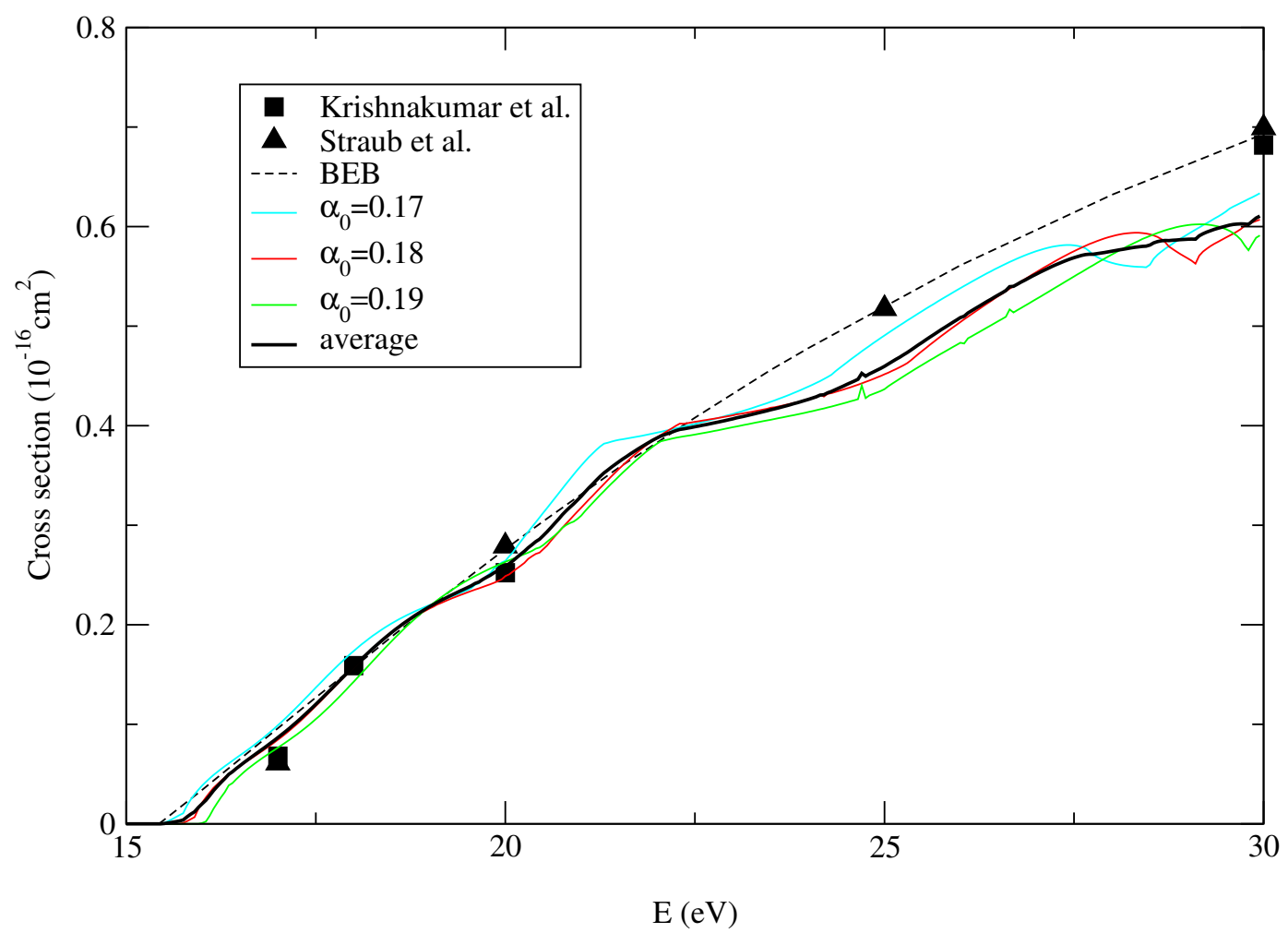

Figure 6. Total ionisation cross section for $\mathrm{H}_{2}$. Dark full line corresponds to the averaged cross section and the light full lines to the $\alpha_{0}$ indicated in the figure. Dashed line corresponds to a BEB calculation (Kim and Rudd 1994). Experimental results: squares, Krishnakumar and Srivastava (1994) and triangles, Straub et al. (1996). 